\title{
THE COLLABORATIVE VIRTUAL MAINTENANCE SYSTEM BASED ON SPNSS
}

\author{
Li Bin \\ Naval University of Engineering \\ Department of Mechanical Engineering \\ Wuhan, China \\ 1021714314@qq.com \\ Li Zhengmin \\ Naval University of Engineering \\ Department of Mechanical Engineering \\ Wuhan, China
}

\author{
Cui Hanguo (Professor) \\ Naval University of Engineering \\ Department of Mechanical Engineering \\ Wuhan, China \\ cuihanguo@163.com, China \\ Cui Jiyue \\ Naval University of Engineering \\ Department of Mechanical Engineering \\ Wuhan, China
}

\begin{abstract}
To realize multiuser collaborative virtual maintenance training of large complex equipment and solve the collaborative work problems such as parallel and competition for resources, a method based on synthesis of Petri nets sharing a kind of subnet for modeling collaborative maintenance process was put forward and the key technologies such as hierarchical modeling of the simulation task, virtual prototype modeling, maintenance process modeling, visual simulation and the program driver were realized. Through the analysis of the functional requirements of the system and based on the humanmachine interaction of the virtual reality development EON Studio function node and the integrated communication function between EonX and Visual Studio, a system framework is presented for multiuser collaborative virtual maintenance simulation training platform and the multiuser collaborative virtual maintenance simulation training system was developed. Take the main power device of a type of ship gear box fault bearing maintenance conversion as an example, we illustrate the working process of the collaborative virtual maintenance system and verify the key technologies of the system.
\end{abstract}

Keywords—collaborative; process modeling; EON

\section{INTRODUCTION}

Collaborative virtual maintenance technology is on the basis of computer, network and virtual reality technology and unites the various maintenance simulation together which scatters in different locations and runs on a different computer platforms through the LAN connection. By means of sharing a realistic virtual environment makes the maintenance personnel complete a variety of complex training mission and have a real-time, synchronous collaborative discussion and interaction design. In recent years, the cooperative problem that computer supported has been widely concerned by domestic and foreign scholars [1-2] .Although many scholars in different fields have made many achievements in the research of cooperative problems, the collaborative virtual maintenance system for supporting multi person parallel operation and its process modeling research are limited[3].
Virtual maintenance simulation process is that in a virtual reality environment, through the computer to complete the repair operation of virtual human interactions between objects and virtual maintenance.

Virtual maintenance simulation process is to achieve the interactive behavior of maintenance operation between virtual person and virtual maintenance objects in the virtual reality environment. The interactive behavior of maintenance operation between virtual person and objects mainly includes human behavior and object behavior. Person's behavior refers to the position, attitude and other characteristics of the virtual person at different times. Object behavior refers to the location of each component to be removed at different times. The interaction between people and objects is usually controlled by "event driven" approach, that is, a maintenance event consisting of a number of maintenance operations, and a maintenance task is composed of multiple maintenance events. Petri network is widely used in the process of modeling. In this paper, Petri net is used as a modeling tool and addressed a method based on synthesis of Petri nets sharing a kind of subnet (SPNSS) for modeling collaborative maintenance process. First, the maintenance sub model of the maintenance personnel is established, and then synthesized to obtain the overall system model.

\section{THE COLLABORATIVE MAINTENANCE PROCESS} MODELING BASED ON THE SYNTHESIS OF PETRI NETS SHARED SUBNET

Petri nets describe the system model through directed graph composed of the node and the arc. It is a system of mathematical and graphical modeling analysis tool and a kind of analysis method to well express the maintenance disassembly process. It not only has the strict mathematical expression form, but also has the visual image representation. The synthesis operation of Petri network is an important way for complex system process modeling, which is widely used in the process modeling of complex systems. Synthetic operation refers to combining all the subsystems with the formation of 
complex systems in a certain way so as to achieve the purpose that the nature of the synthesis system is replaced by the nature of the subsystem. The synthesis of Petri net system is divided into Shared synthesis and simultaneous synthesis. Sharing synthesis can realize the effective sharing of resources and reduce the excessive occupation of resources which is reflected in the virtual human resources, virtual maintenance and virtual tools and so on in the collaborative maintenance. Synchronous synthesis is mainly used for the concurrent synchronization of complex systems [4]. In this paper, a kind of improved synthesis method is proposed, which is based on the sharing of SPNSS and which can realize the sharing of the resources and realize the concurrent operation.

\section{A. Basic definition of SPNSS}

The basic definition of the related concepts of Petri net is defined as follows.

Definition 1: Two Petri nets structure $N=(P, T, F, W)$ and $N_{1}=\left(P_{1}, T_{1}, F_{1}, W_{1}\right)$, if satisfied:

(1) $P_{1} \subset P, T_{1} \subset T$ and $P_{1} \neq \phi, T_{1} \neq \phi$;

(2) $F_{0}=F \cap\left(\left(P_{1} \times T_{1}\right) \cup\left(T_{1} \times P_{1}\right)\right)$;

Then $N_{1}$ is called a subnet of $N$.

Definition 2: Let $\mathrm{t} N_{i}=\left(P_{i}, T_{i}, F_{i}, W_{i}\right)(\mathrm{i}=1,2)$ to be two petri nets system, if satisfied:

(1) $P=P_{1} \cup P_{2}, P_{0}=P_{1} \cap P_{2} \neq \Phi$;

(2) $T=T_{1} \cup T_{2}, T_{0}=T_{1} \cap T_{2} \neq \Phi, F=F_{1} \cup F_{2}$;

(3) $\forall x, y \in P \cup T: W(x, y)=W_{1}(x, y)+W_{2}(x, y)$;

(4) $N_{1}$ and $N_{2}$ shared the subnet of $N$.

Then Nis one SPNSS net of $N_{1}$ and $N_{2}$.

Definition 3 : $\quad \sum_{i}=\left(N_{i}, M_{0 i}\right)(\mathrm{i}=1,2)$ : Petri net system, if system $\sum\left(N, M_{0}\right)$ satisfied:

(1) $N$ is one SPNSS net of $N_{i}$;

(2) $M_{0}(p)=\left\{\begin{array}{l}M_{10}(p) \forall p \in P_{1}-P_{0} \\ M_{20}(p) \forall p \in P_{2}-P_{0} \\ M_{10}(p)+M_{20}(p) \forall p \in P_{0}\end{array} ;\right.$

Then $\sum\left(N, M_{0}\right)$ is one SPNSS system of $\sum_{i}=\left(N_{i}, M_{0 i}\right)(\mathrm{i}=1,2)$.

\section{B. The Characteristics Analysis of SPNSS}

According to the different initial state identification, structure and parameters, the Petri net exhibit different characteristics in describing the system, so the characteristics of the Petri net should be analyzed in order to evaluate the characteristics of the system. According to the different initial identification, the characteristics of the system are divided into behavior and structure. The initial identification $M_{0}$ is different, and then the system behavior characteristic is different. The typical behavior characteristic includes the system's reachability and activity. The structural characteristics of the system such as boundedness, safety and so on are independent of the initial identification.

The Petri net is active which means that in any state the activation of each change can be triggered by the appropriate trigger.

It is necessary to analyze the structural activity of Petri net, which is the necessary condition for the synthesis of the net to keep the activity of the synthesized network.

During the synthesis of Petri nets Shared subnet, it is necessary to analyze the structural activity of Petri net, which is the necessary condition for the synthesis of the net to keep the activity of the synthesized network. If the changes of $n$ network system represented by $\sum_{i}=\left(N_{i}, M_{0 i}\right)(\mathrm{i}=1,2 \cdots n)$ can be triggered by any trigger, the change of the synthetic network system $\sum\left(N, M_{0}\right)$ is also triggered by any trigger conditions.

\section{The process model of collaborative maintenance based on SPNSS}

In real systems, when two or more subsystems share the same nature of resources (such as virtual maintenance, maintenance tools, etc.) and each subnet maintain the structural activity, each system's change have the same rule and trigger in the same time, the Petri nets system can be synthetized and the synthetic network system still maintain the structure activity 56-57.

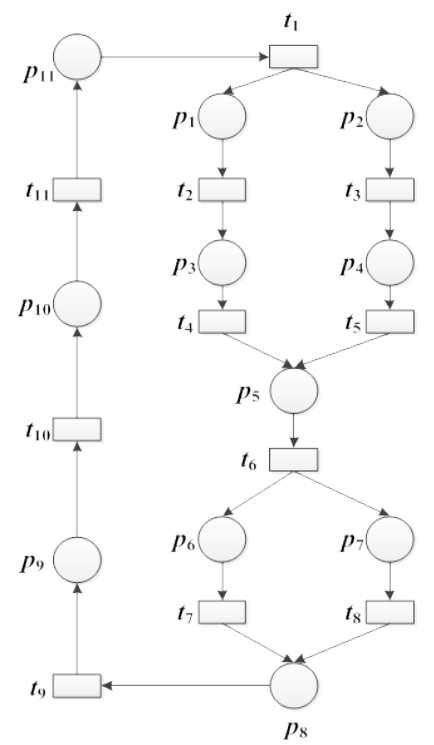

Fig. 1. Submodel $N_{1}$ of maintenance Petri net 
In collaborative virtual maintenance conditions, numerous maintenance staffs disassembly and maintenance on the same operation object through the network at the same time in different places and computer terminal. Since the operation of individual users is unknown and the shared resources (especially for the non-consumption of resources, such as the limits of imaginary personification, tools) are limited, the detachability of parts may be affected by conflict between the independent behaviors of user. Inappropriate dispose will block the process of maintenance disassembly and cannot simulate the real maintenance training scenarios. Accordingly, we need to analyze the process of maintenance and establish a complete control and optimization method. Model the cooperative maintenance system by using the SPNSS process modeling method. First, the maintenance sub model of the maintenance personnel is established, and then the maintenance sub model is synthesized. In the case of collaborative maintenance, maintenance personnel $\mathrm{A}$ and maintenance personnel $\mathrm{B}$ use the same maintenance resources (the same repair tool) to replace the faulty parts of the maintenance equipment, and reassemble the equipment as an example, apply the method of SPNSS for system modeling. Specific steps are as follows:

Step 1: According to the constraints of equipment parts, establish each sub model of maintenance Petri nets.

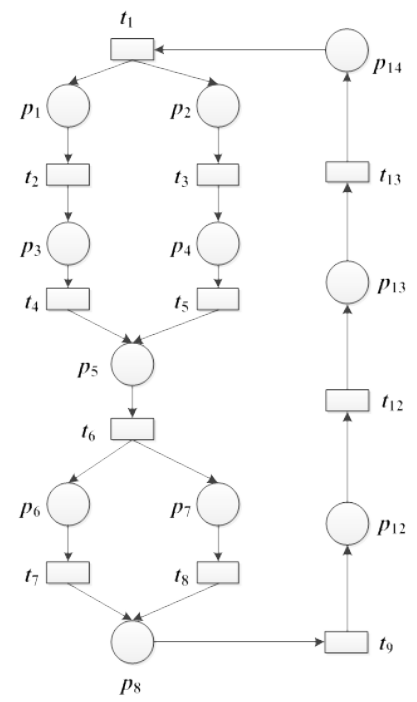

Fig. 2. Submodel $N_{2}$ of maintenance Petri net

Figure 1 is the subnet model of Petri net $N_{1}$ and Figure 2 is the subnet model of Petri net $N_{2}$ and the $\mathrm{T}$ and $\mathrm{P}$ have the following meanings:

$t_{1}$ :Start maintenance; $p_{1}$ :Part $1 ; t_{2}$ :Disassembly parts; $p_{2}$ : Part $2 ; t_{3}$ : Disassembly parts; $p_{9} 、 p_{12}$ :The fault parts to be removed; $t_{9}$ : Disassembly the fault parts; $p_{10}, p_{13}$ :The replacement part 9; $t_{10}$ : Replace the faulty parts;; $t_{11}$ : Assembly parts; $p_{11} 、 p_{14}$ :The equipment (completed maintenance and disassembly).

Step 2: If $N_{1}$ and $N_{2}$ shares a subnet, then determine whether the following formula are established:

$$
\begin{aligned}
& \forall x, y \in P \cup T: W(x, y)=W_{1}(x, y)+W_{2}(x, y) \\
& W(x, y)=W_{1}(x, y)+W_{2}(x, y)=\left\{\begin{array}{cccccccccccccc}
0 & 2 & 0 & 0 & 0 & 0 & 0 & 0 & 0 & 0 & 0 & 0 & 0 & 0 \\
0 & 0 & 2 & 0 & 0 & 0 & 0 & 0 & 0 & 0 & 0 & 0 & 0 & 0 \\
0 & 0 & 0 & 2 & 0 & 0 & 0 & 0 & 0 & 0 & 0 & 0 & 0 & 0 \\
0 & 0 & 0 & 0 & 2 & 0 & 0 & 0 & 0 & 0 & 0 & 0 & 0 & 0 \\
0 & 0 & 0 & 0 & 0 & 2 & 0 & 0 & 0 & 0 & 0 & 0 & 0 & 0 \\
0 & 0 & 0 & 0 & 0 & 0 & 2 & 0 & 0 & 0 & 0 & 0 & 0 & 0 \\
0 & 0 & 0 & 0 & 0 & 0 & 0 & 2 & 0 & 0 & 0 & 0 & 0 & 0 \\
0 & 0 & 0 & 0 & 0 & 0 & 0 & 0 & 1 & 0 & 0 & 1 & 0 & 0 \\
0 & 0 & 0 & 0 & 0 & 0 & 0 & 0 & 0 & 1 & 0 & 0 & 1 & 0 \\
0 & 0 & 0 & 0 & 0 & 0 & 0 & 0 & 0 & 0 & 1 & 0 & 0 & 1 \\
2 & 0 & 0 & 0 & 0 & 0 & 0 & 0 & 0 & 0 & 0 & 0 & 0 & 0
\end{array}\right\}
\end{aligned}
$$

$\mathrm{W}$ represents the input function from the place $P$ to the change $T$.

Step 3: The synthesis of $N_{1}$ and $N_{2}: N=N_{1} \cup N_{2}$.

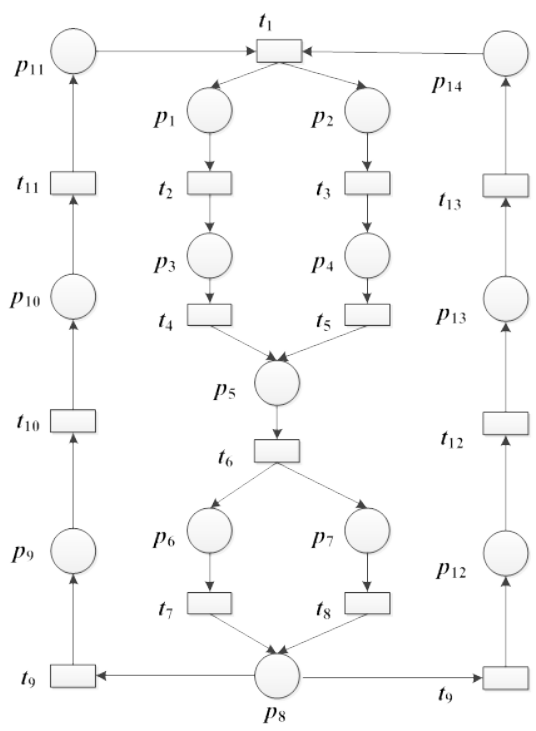

Fig. 3. Synthesis of Petri nets $N$ sharing a kind of subnet

Because the maintenance personnel 1 and 2 share the same maintenance tools of the same working table to replace the faulty parts in the collaborative maintenance of virtual environments, so we can use the SPNSS synthesis method. The synthesis net $\mathrm{N}$ is as follows. Hence through the synthesis method SPNSS can achieve the effective sharing of resources, and can achieve the concurrent operation.

\section{COLLABORATIVE MAINTENANCE PROCESS SIMULATION IN EON ENVIRONMENT}

\section{A. Disassembly Process Planning of Products Based on SPNSS}

Main power devices mainly consists of marine diesel engines, gear box, power of shafting and propulsion system. In the follow paragraph only take the gear box below as an example. Fatigue wear and tear, improper design and maintenance of poor reasons can lead to the operation fault of 
the gear box, what is more common is bearing, shaft and pump failure among them. With the proficiency maintenance changing of the breakdown of the gearbox bearing is one of the main Through the mechanical design module and the human body builder module of three-dimensional modeling software CATIA to establish a gear box, maintenance tools and localization of the virtual human mode respectively. Using the process modeling method SPNSS to model the cooperating maintenance system of replacing the gearbox's fault bearing.First of all, we should analyze the behavior and structure characteristics of the process model. As the structural characteristics of the maintenance simulation are independent of the initial identification and all parts are removable, only the correlation matrix and the state equation of behavior characteristics are analyzed.

Although the correlation matrix can be obtained by calculation, the gearbox parts quantity is a sizable one and the matrix dimension is too high, so the paper uses the simulation software PIPE to generate the correlation matrix directly. After modeling, click the menu bar on the left side of the modules available modules, select incidence\& marking, double-click the default and then click on the new window is the associated matrix of the Petri net.

The state equation of Petri net is :

$$
M(k)=M(k-1)+C^{T} u_{k}, k=1,2 \cdots
$$

According to the constraint relationship of the arc, the assembly sequence of the bearing is obtained. $\left(t_{16}, t_{17}\right),\left(t_{14}, t_{15}\right), t_{13},\left(t_{11}, t_{12}\right)\left(t_{9}, t_{10}\right),\left(t_{7}, t_{8}\right), t_{6}\left(t_{4}, t_{5}\right),\left(t_{2}, t_{3}\right), t_{1}$

\section{B. Identify the Headings}

The virtual maintenance scenarios of this system and the 3 $\mathrm{d}$ visualization model of interaction are achieved through the virtual reality software EON Studio's drive and routing mechanism. Through the ClickSensor node receive the user instruction and the Task and the Latch node control the parts disassembly. The system can read remove the next node in the list, so as not to cause all parts at the same time remove the phenomenon. Record the number of error remove operation using the Counter node. The part routing diagram and simulation of collaborative virtual maintenance are as shown below.

Private void myEon_OnEvent(object sender, AxEONXLib2._IControlEvent_OnEventEvent e)

$\{$ if (e.bstrNodeName == "')

return;

cmdmsg.NodeValue = e.pvarNodeValue; if (

this.formManager.NetManager.SendGroupMsg(cmdmsg) $==$ false)
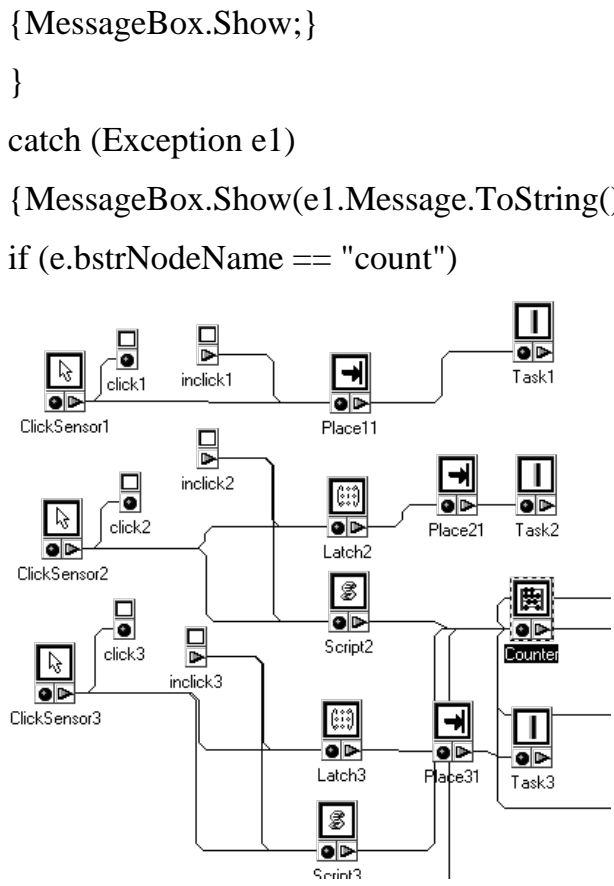

Fig. 4. The routing figure of collaborative virtual maintenance

\section{CONCLUSIONS}

Based on the introduction of Petri net, the paper presents the analysis model of Petri net (SPNSS) for collaborative maintenance process modeling, and describes the basic definition, structure characteristics and behavior characteristics of the model.

\section{REFERENCES}

[1] Moreura L O, SOUSA F R C, MACHADO J C. A distributed concurrency control mechanism for XML data [J]. Journal of Compute and System Science, 2011， 38(9): 1009-1022

[2] Yuan Jing. Research on cooperative awareness in CSCW system [J]. Computer applications and software, 2008,25 (8) : 207-208

[3] Chao Zhao , Xing Xin Li, Kong Xia. Communication design of collaborative virtual maintenance training system Based on JMS[J]. Applied Mechanics and Materials, 2014, 556(5):4871-4874

[4] J.L.Peterson . Petri net theory and the modeling of systems [J]. Information Fusion, 2012, pp. 48-59. 